\title{
Estéticas enraizadas: aproximaciones al video indígena en América Latina
}

\section{Rooted Aesthetics: Approaches to Latin American Indigenous Media}

\section{Amalia Córdova}

Smithsonian National Museum of American Indian

fvc@si.edu

\section{Resumen}

Este ensayo examina las formas en que el audiovisual de origen indígena es producido y circulado en América Latina, ofreciendo una discusión sobre los conceptos claves y definiciones que se le ha dado a este cuerpo de obra, y sobre sus prácticas específicas.

\section{Palabras clave}

Video indígena, cine latinoamericano, comunicación indígena.

\section{Abstract}

This essay looks at the ways indigenous-produced audiovisual media is produced and circulated in Latin America, offering a discussion of some of the key terms and definitions placed on this body of work, as well as its distinctive practices.

\section{Keywords}

Indigenous media, Latin American cinema, indigenous communications. 
"En realidad no soy un realizador independiente. Aunque la cuestión técnica para realizar un video la haga solo, el sentir y el contenido de mis videos pertenecen al pueblo." Mariano Estrada Aguilar (Córdova, 2004)

"[... hemos llegado a un momento en el que decimos, ... pues hagamos cosas más de... nuestros sentimientos, de expresión más individual." Carlos Pérez Rojas (Zamorano, 2004).

\section{El "video indígena" en América Latina}

Cuando se habla de cine latinoamericano, se tiende a pensar en largometrajes premiados en grandes festivales, en el documental histórico, o en cortos experimentales de innovación técnica. Sin embargo, son pocos quienes imaginan que todas estas expresiones audiovisuales también se están produciendo desde una perspectiva indígena, dentro de una corriente que se podría llamar "video indígena," y de gran impacto para el movimiento social por los derechos indígenas.

A partir de la década del setenta, las comunidades indígenas en las Américas o Abya Yala han incursionado en estos nuevos medios, desde la implementación de radios comunitarias y el uso de Internet hasta la producción de largometrajes en sus propias lenguas. Desde reportajes a animación, pasando por videos musicales, video-cartas, obras experimentales, ficciones y diversas formas híbridas del documental, los productores indígenas han adaptado nuevas tecnologías de comunicación ${ }^{2}$-en especial el video-para crear obras de gran alcance que desafían el persistente mito de que los pueblos indígenas han desaparecido o que carecen de capacidad para tomar el control de su imagen.

En América Latina, este movimiento ha ido creciendo con el tiempo, y tanto su práctica como su obra se ha dado a conocer como "video indígena," término empleado por el Instituto Nacional Indigenista en México en 1991 (Wortham, 2004), “vídio Índio" (usado informalmente en Brasil) o "medios indígenas" (del inglés “indigenous media," según la antropóloga Faye Ginsburg, 1991). Estos videos aportan a la visibilización y a la participación política de los pueblos indígenas u originarios, incidiendo en luchas lingüísticas, legales y culturales. A través de video, los comunicadores indígenas logran documentar su memoria histórica y el acontecer actual, plasmar sus saberes y costumbres, educar a los jóvenes en las

\footnotetext{
1 Abya Yala es un término kuna que designa el continente americano, y es usado ampliamente en contextos de política indígena.

2 El uso de estas nuevas tecnologías por parte de comunidades periféricas, subalternas o marginales se ha trabajado ampliamente en estudios sobre los medios comunitarios y las llamadas (en inglés) "information and communication technologies" (ICTs) o tecnologías de información y comunicación.
} 
tradiciones y lenguas, y fortalecen la identidad comunitaria en una compleja realidad contemporánea.

Se trata de un corpus de obra bastante heterogéneo, geográficamente disperso, y ligado fuertemente a procesos sociales y presiones locales. Gran parte de estas obras se desarrolla incorporando valores, protocolos y metodologías de cada comunidad o pueblo indígena, lo que Ginsburg ha denominado "embedded aesthetics," lo que se traduce como estéticas incrustadas o enraizadas, que denotan el modo de producción de las obras, determinando los procesos de producción así como los productos mismos (Ginsburg, 1994). Los realizadores indígenas a menudo navegan agitadas corrientes políticas locales, regionales y nacionales (entiéndase estadonación), y a su vez intentan mantener un grado de inserción y diálogo con la vida en sus comunidades de origen, con sus propias luchas y presiones internas. La mayoría de los colectivos que realizan proyectos de comunicación indígena luchan constantemente por sostenerse y manejan delicadas relaciones con agencias nogubernamentales de derechos humanos o de defensa de los derechos indígenas, y se mantienen en alerta ante constantes amenazas y atropellos a sus derechos en el día a día. Por estos motivos es difícil (pero no imposible) que surjan grandes apuestas personales al estilo del cine de autor.

Como marco de referencia, incluso “América Latina” resulta un término complicado e impreciso en sí mismo, ya que evoca la idea de un continente conformado por estructuras políticas de estado-nación, las que mantienen un constante asedio hacia los territorios tradicionales de las naciones indígenas que a menudo difieren históricamente de las fronteras colonialmente determinadas. Dentro de las estructuras de estado-nación existen naciones indígenas soberanas que no se identifican con el proyecto de nación post-colonial; en algunos casos se trata de reservas físicas, como resguardos o reducciones, sujetos a permanente acoso e intervención del estado, en otros casos se trata de poblaciones desplazadas o profundamente afectadas por la migración a grandes ciudades o al extranjero, adonde difícilmente van a identificarse (o conseguir reconocimiento) como "indígena americano," "latino" o "hispano, " y adonde la forma tradicional de vida comunitaria se ve gravemente interrumpida. Así, el debate sobre "lo indígena” está siempre en curso y aflora con frecuencia en el contexto del llamado "video indígena."

Se pueden identificar algunos rasgos de filiación en las obras que representan al video indígena en los festivales de cine indígena pero no constituyen un género audiovisual propiamente tal; la idea del género audiovisual, como estrategia de mercadeo para obras en un circuito de consumo, difícilmente puede albergar obras tan disímiles, de proveniencia y factura muy variada, que resiste la imposición de estándares industriales. Tampoco se puede calificar toda obra de temática indígena 
como "video indígena." Como veremos a continuación, se trata de obras improbables que irrumpen en un paisaje audiovisual dominado por legados coloniales, y de redes de solidaridad que las ayudan a circular.

\section{Desgranando el "video indígena" ${ }^{3}$}

Para el público poco familiarizado con las obras de producción indígena, el primer acercamiento puede ser un proceso inquietante, pues choca con suposiciones fundacionales de la educación occidental, adonde lo indígena está relegado a una historia pasada, considerado exterminado, estático en el tiempo o consagrado como objeto de archivo etnográfico. Estas percepciones de lo indígena como algo extemporáneo, caduco y folclórico, son recicladas en un loop sin fín por los medios de comunicación masivos. Además, no se trata de obras que sean fáciles de describir en una sola frase; el público en general a nivel mundial carece de referentes reales para ubicarse en el imaginario indígena. Los realizadores indígenas han esperado un largo tiempo para comunicarse a través de su trabajo, y no necesariamente van a hablarle al público en general, ni en su lengua; tal vez el mensaje se codifica para circular principalmente dentro de la propia comunidad. Y como la tradición oral está muy viva en las comunidades indígenas, el hablar no debe ser apresurado o interrumpido, y las obras pueden tomarse su tiempo en desarrollar sus mensajes.

Varias preguntas circulan en torno al llamado video indígena, incluso para sus practicantes, y quienes lo apoyan y lo difunden: ¿cómo se visualiza el video indígena a largo plazo? ¿a quién interpela? ¿Qué beneficios o resistencias ofrece este término? El rótulo de "video indígena" ¿empodera o margina a los realizadores? ¿Como categoría, qué tanto acomoda las confluencias estilísticas, las colaboraciones inter-y trans-culturales, y las anomalías, como el mestizaje, la colaboración inter-étnica, y la autoría colectiva? ¿Cuáles son los modos particulares de producción, los discursos y las formas de circulación que el video indígena ha engendrado?

Desde su surgimiento en México en 1991, diversas definiciones del video indígena se han planteado desde la perspectiva académica. Cabe señalar que gran parte de la escritura académica sobre los medios indígenas ha transcurrido desde sus inicios principalmente en inglés desde universidades norteamericanas, como el trabajo de Patricia Aufderheide sobre Video nas Aldeias en Brasil (Aufderheide, 1995) y desde el foco disciplinario de la antropología visual, comenzando con Terence Turner y Mónica Frota, quienes escriben sobre el uso de video del pueblo Kayapo en Brasil (Turner, 1990, 1991, 1995 y 2002, y Frota, 1993). Una de las pocas autoras que ha publicado regularmente en forma bilingüe es la académica

3 El título de esta sección está inspirado en el artículo de Wortham (2004), que habla de "desempacar" el video indígena. 
Freya Schiwy, conocida por su estudio del video indígena en los Andes como elemento de descolonización (Schiwy, 2002, 2009). Los teóricos Ella Shohat y Robert Stam también han publicado su tratado multi-disciplinario Unthinking Eurocentrism (1994) en varios idiomas, citando entre otros el trabajo del proyecto Video nas Aldeias en Brasil como un desafío a la ideología colonial de los medios dominantes.

Sin embargo, es la antropóloga Erica Wortham (2004) quien abre la teorización en torno al término de video indígena puntualmente en el contexto mexicano: "al igual que los términos nativos, indígenas y originarios, el video indígena se ha apropiado y re-significado conscientemente como una postura o posición política fundamental para las luchas indígenas por la autodeterminación.” La directora y fundadora del Chiapas Media Project (Promedios de Comunicación Comunitaria), Alexandra Halkin, prefiere utilizar el término "video controlado por indígenas" (“indigenously-controlled video," en Halkin, 2008). A su vez, la antropóloga Claudia Magallanes-Blanco (2008) propone un marco dialógico Bakhtiniano para describir documentales producidos sobre la rebelión zapatista en México, colocando el trabajo de Promedios junto a un proceso de video independiente sobre el movimiento Zapatista, y no segregándolo como video indígena.

Alvear y León resumen la propuesta de Schiwy (2002) para distinguir el video indígena de otras formas de cine clásico:

a) está sujeto a un régimen de producción colectivo y no-especializado, b) es producido directamente por indígenas, c) no está dirigido al mercado, d) está sujeto a un régimen de propiedad e intercambio no-capitalista, e) es un relato no intelectual ni experimental que juega libremente con los géneros cinematográficos y audiovisuales (Alvear y León, 2009:109).

Lineamientos tan específicos pueden resultar útiles para categorizar tipos de cine, pero el universo audiovisual de los pueblos indígenas es mucho más variado. Juan José García, director zapoteca que trabaja en México desde la década de los noventa, aclaró en un diálogo con Wortham: "El vídeo indígena no existe... tal vez existe lo que se podría llamar 'video zapoteca', o 'video Juan José', pero el video indígena es más una postura que un género audiovisual claramente diferenciado” (Wortham, 2005:7). Con esta astuta observación, García complica la homogeneización de los videos que se generan en los proyectos estatales en México, y cuestiona del uso de esta categoría como rasgo principal. Los debates acerca del video indígena al final se tratan más de las expectativas que genera este término y de quién se beneficia de él. Complementando la citas que abren este ensayo, la observación de García propone un espacio para que él se considere un director ("video Juan José"), y no sólo un colaborador, al mismo tiempo señala la necesidad de reconocer su afiliación a su comunidad ("video zapoteca”). Para García, una 
categoría tan amplia como video indígena no acomoda estas funciones polivalentes, que no tienen porqué contradecirse.

La red de organizaciones de cine y video indígena alineada con la Coordinadora Latinoamericana de Cine y Comunicación de los Pueblos Indígenas (CLACPI) publicó su propia definición (colectiva y anónima) del video indígena:

Para las organizaciones y los individuos que componen CLACPI, el cine y/o video indígena incluye obras, y sus directores y cineastas, que aplican un firme compromiso de dar voz y visión digna del conocimiento, la cultura, proyectos, reclamos, logros y luchas de los pueblos indígenas. Implícita también está la idea de que este tipo de cine y video requiere un alto grado de sensibilidad y la participación activa de las personas que aparecen en pantalla. Dicho de otro modo, el cine y video indígena intenta utilizar esta poderosa herramienta para fomentar la auto-expresión y fortalecer el desarrollo real de los pueblos indígenas. (CLACPI, 2009).

Esta definición va dirigida al mundo exterior, y su publicación evidencia la opacidad en torno a lo que se entiende como cine y video indígena. Aquí, el cine o el video indígena se presenta principalmente como una cualidad que se distingue por las buenas prácticas (consulta, trato y representación respetuosa de los pueblos indígenas), haciendo hincapié en la agencia y la voz de los pueblos indígenas en las obras, y restando importancia a las especificaciones técnicas o los roles puntuales en la producción ${ }^{4}$.

El enfoque de CLACPI de resaltar las luchas de los pueblos originarios se remonta a las luchas de los diferentes movimientos sociales y del cine de los años sesenta y setenta, como el Tercer Cine, que alinea los diversos cines mundiales desde su marginalidad y situación periférica con respecto al primer mundo y su cine comercial-un "primer" cine- y adonde se considera el cine de autor un "segundo" cine (Solanas y Getino, 1969). La capacitación de realizadores indígenas comenzó de distintos modos en diferentes lugares, creciendo hacia un proyecto continental, trans-originario con aspiraciones des-colonizantes, y ha crecido hasta abarcar una comunidad cada vez más global y amplia que incluye el audiovisual de poblaciones originarias de Europa, el Ártico, Asia y Africa, afro-descendientes, desplazados y otras minorías o grupos marginados de proyectos nacionales.

Los movimientos sociales del cine latinoamericano y sus manifiestos como el "Cine Imperfecto" (García Espinosa, 1969), "La Estética del Hambre” (Rocha, 1965) y el Cine Revolucionario liderado por directores como Jorge Sanjinés resonaron dentro de las élites nacionales culturales en sus intentos de representar la

\footnotetext{
4 Esta posición varía de otros festivales y organizaciones de cine, como el ImagiNative Film + Media Arts Festival en Toronto, un festival de cine originario que sólo acepta obras dirigidas, escritas o producidas por realizadores indígenas, sin importar la temática.
} 
realidad latinoamericana sin exclusiones, situando en primer plano la clase obrera, los campesinos y los oprimidos. El indigenismo históricamente ha ocupado un espacio importante en la creación y reinvención del proyecto de estado-nación, por lo que para muchos cineastas, la resistencia de los pueblos originarios se convirtió en ícono para la lucha de clases o para proyectos revolucionarios ${ }^{5}$. La mayoría de las películas indigenistas buscaban solidarizar con los pueblos indígenas, representando sus luchas aunque poco se permitió que tuvieran roles decisivos en la producción de las obras ${ }^{6}$.

La invisibilización de los pueblos indígenas contemporáneos en el cine de América Latina y en las pantallas de televisión también crea una presión histórica para los comunicadores indígenas. El cine y video indígena siguen en tensión con el archivo fílmico mundial, que desde su origen fomenta el exotismo de los pueblos indígenas, tanto en cine etnográfico como narrativo, producido localmente o por equipos extranjeros. Algunos comunicadores indígenas han comenzado a abrir, repatriar y reprocesar sus archivos para contar sus propias historias de una manera muy poderosa y conmovedora, especialmente visible en trabajos de Video nas Aldeias de Brasil, como Xina Bena/Nueva Era (2006), Pirinop: Mi primer contacto (2005/2007), De Volta a Terra Boa/Volver a la buena tierra (2008) y Ja Me Transformei em Imagem/Ya me convertí en imagen (2008). En estos documentales, las comunidades revisan y comentan material filmico de archivo, recreando y contando su versión de situaciones pasadas, y exponiendo estos hechos para las nuevas generaciones con un contexto propio.

Para ayudar a la difusión de estas importantes obras, han surgido importantes espacios en festivales internacionales de cine, $y$ se han creado festivales especialmente abocados al cine indígena. Éstos se convierten en eventos emblemáticos que hacen visible una comunidad global de video indígena, que reúnen obras y productores geográficamente muy dispersos, generando espacios fértiles para el debate sobre los derechos indígenas y la representación, abriendo fuentes de apoyos e intercambios, facilitando la circulación de obras devuelta a las

\footnotetext{
5 En Bolivia, por ejemplo, los cineastas Jorge Sanjinés y Jorge Ruiz fueron entre los primeros en abordar las luchas de los pueblos indígenas con finalidades de concientización social y política. Sanjinés y el Grupo Ukamau abrieron los senderos para un cine indigenista sudamericano, trabajando con indígenas que no eran actores profesionales como el director aymara Reynaldo Yujra, e investigando de modos alternativos y no- comerciales de circulación (Sanjinés, 1979). En Argentina, la obra temprana de Jorge Prelorán daría cuenta de realidades indígenas ante un país que niega terminantemente su población indígena, y en Perú, Federico García Hurtado escribe y dirige el largometraje Tupac Amaru (1984) sobre la vida del guerrero andino Tupac Amaru, calificando su lucha como "la primera revolución social e independentista de América."

6 Schiwy (2009) profundiza en las distancias que tiene el video indígena en la región andina con éstos movimientos.
} 
comunidades indígenas y aún más allá. Se trata de una red de solidaridad comprometida con los contenidos y los realizadores de estos videos.

\section{Los lazos con los movimientos indígenas}

El movimiento por la auto-representación y por el derecho a la comunicación indígena nace junto la historia de resistencia y defensa de los derechos de las comunidades originarias. Desde la década de 1970, las luchas por la autodeterminación de los pueblos indígenas de América se unieron en torno a temas cruciales como los derechos humanos y territoriales. La organización regional de los movimientos indígenas de distintos países desde la década de los sesenta a los ochenta fueron cada vez más reconocidos por parte de los sectores políticos luchando contra dictaduras. Comenzaron a organizarse las movilizaciones contra el tono de celebración del quinto centenario del contacto europeo. Varios frentes regionales indígenas se organizaron en la década del ochenta, dando paso a la construcción de un movimiento social indígena continental que vio nacer importantes reuniones y declaraciones, como el Grupo de Trabajo de Asuntos Indígenas las Naciones Unidas creado en Ginebra, que veinte años después se traduce en el Foro Permanente de Asunto Indígenas y en la adopción de las Naciones Unidas de la Declaración de los Derechos de los Pueblos Indígenas en septiembre de 2007 en Nueva York.

En 1992, junto con actos oficiales de conmemoración del contacto europeo, ocurrieron contra-manifestaciones que rechazaron el tono romántico y festivo de la época, exigiendo un replanteamiento de la historia y de la situación actual de los pueblos indígenas (ver el "Debate de Colón" y Quinto Centenario, en Shohat y Stam, 1994). Las protestas de 1992 provocaron reuniones internacionales, innumerables declaraciones, y en particular en las Américas, abrieron un profundo cuestionamiento de las identidades nacionales. Quizá la más poderosa y visible encarnación de este movimiento fue el levantamiento Zapatista en el estado mexicano de Chiapas, logrado con el despliegue estratégico de los medios de comunicación, incluyendo producción audiovisual indígena e independiente (Magallanes-Blanco, 2008). Este levantamiento se convirtió en un hecho emblemático que llevó "la cuestión indígena" al centro de atención nacional e internacional, con la ayuda de una fuerte estrategia comunicacional que involucró el uso de Internet y la elaboración de un cuidado discurso, emanado poéticamente del rostro velado del sub-comandante Marcos.

Hoy existen distintos niveles de organización indígena con procesos particulares, tanto locales, regionales y nacionales, en diálogo con otros organismos internos, partidarios y externos. Existen organizaciones creadas por los propios indígenas, las creadas por el estado, las organizaciones religiosas y de la iglesia católica, y las que 
son creadas por personas o fundaciones no-indígenas. No todos los casos son iguales en cada región, son diferentes procesos de organización que han logrado consolidarse con mayor o menor estabilidad. En el proceso de organización existen instituciones tradicionales y ciertas formas de apropiación de sistemas de organización española, como los cabildos. Existen los cacicazgos, el matriarcado, los clanes, y otras identificaciones como grupo étnico, los que se pueden conformar en bases locales para avanzar a organizaciones regionales, zonales o nacionales.

Otras formas de organización, como el Consejo Regional Indígena del Cauca (CRIC) en Colombia, nacen por inconformidad local, generando encuentros de los cuales surgen organizaciones, o bien las luchas se unifican, como la lucha ante la discriminación, los terratenientes, o la pérdida del territorio, entre otras. En otros casos la organización surge más bien de procesos regionales o nacionales, como el caso de la Confederación de Nacionalidades Indígenas del Ecuador (CONAIE) en Ecuador, y diversas confederaciones en Bolivia y Perú. En síntesis, se podría decir que algunos elementos aglutinantes son la lucha por la defensa y recuperación el territorio; la lucha contra la discriminación social, de parte del estado y de la sociedad civil, y por los derechos de la mujer indígena; la lucha por la recuperación de formas de organización o autoridades tradicionales; y el fortalecimiento de las estructuras y elementos culturales tradicionales.

En 1991, presionado por confederaciones indígenas regionales y nacionales, el gobierno de Colombia sentó un precedente importante con la incorporación de líderes indígenas en la Asamblea Constituyente, logrando la inclusión de los derechos indígenas en la constitución colombiana por primera vez y garantizando cupos indígenas en el Senado. Los gabinetes de comunicación de las organizaciones indígenas más importantes se acercaron a realizadores de cine para encargar obras o hacer videos propios, creando un registro audiovisual del movimiento indígena, y eventualmente la ONIC creó su propio Centro de Documentación. Los cineastas independientes y socialmente comprometidos Jorge Silva y Marta Rodríguez jugarían un papel crucial en la documentación de una historia de genocidio, persecución y resistencia de los pueblos indígenas de Colombia, y son reconocidos por el mismo movimiento indígena de Colombia por su trabajo.

Varias organizaciones indígenas nacionales y regionales como CONAIE, CRIC, ACIN (Asociación de Cabildos Indígenas del Norte del Cauca, en Colombia), y ONIC (Organización de Nacionalidades Indígenas de Colombia) tienen sus propios Departamentos de Comunicación, que se dedican principalmente a publicar comunicados sobre situaciones de violación de derechos y en algunos casos a producir programas radiales que se transmiten por Internet ${ }^{7}$. Sin embargo,

7 Ver la página web del CRIC, que transmite audio en-línea: http://www.cric-colombia.org/. 
no disponen de fondos como para estar produciendo una gran cantidad de material audiovisual. Como señala un ex-dirigente del CRIC, Jesús Avirama:

Para nosotros el video es tan importante como la utilización de agua, y los medios de comunicación como las emisoras, en donde nosotros podamos divulgar los distintos problemas y presentar nuestros puntos de vista con referencia a las soluciones a esos problemas. Tenemos un equipo de comunicaciones el cual esta produciendo materiales sobre todo de capacitación para la misma comunidad. Tenemos muy pocos materiales para divulgar hacia afuera de las comunidades, pero creemos que esta área la tenemos que abordar también. (Benamou, 1994)

Estas organizaciones han tomado en cuenta el valor de la comunicación, participando paulatinamente en reuniones de comunicación indígena, como la primera Cumbre Continental de Comunicación Indígena de Abya Yala (realizada del 8 al 12 de noviembre de 2010 en Cauca, Colombia y reuniendo comunicadores indígenas de más de 25 países del continente) y llevando videos para presentar en grandes encuentros internacionales. Durante la Cumbre, se hizo una pequeña muestra de video indígena en la ciudad de Popayán, aportada y organizada por CLACPI, y las mesas de la cumbre se transmitieron en vivo desde la página web del $\mathrm{CRIC}^{8}$.

\section{Modos imperfectos de producción}

El limitado acceso al equipo y la infraestructura para producir películas es aún un problema para los realizadores originarios. En general se hace uso del género documental, aunque la ficción se hace menos no por elección sino por su elevado costo de producción y por la priorización de sacar a la luz temas de derechos humanos con cierta urgencia. Las producciones suelen ser cortas (20 a 30 minutos), y de presupuesto muy bajo. El formato más usado es el video (HD, miniDV, y en algunos casos VHS), ya que los costos de hacer cine son prohibitivos. Los escasos equipos rotan de mano en mano y son pocos los realizadores que poseen sus propias cámaras o equipos de edición. Sin embargo, las organizaciones y colectivos de comunicación indígena logran gestionar tanto equipos como talleres mediante sus relaciones con organizaciones internacionales sin fines de lucro y organizaciones no-gubernamentales, movimientos de solidaridad, cooperación y de protección medioambiental, y en algunos casos, apoyos estatales.

En su mayoría los comunicadores indígenas no han tenido una capacitación formal en realización cinematográfica, con algunas notables excepciones de

8 La cumbre redactó una declaración que exige el derecho a la comunicación (disponible en http://www.cccia-2010.com/), y se planifica un Segundo Encuentro continental en México para fines de 2012 . 
directores que han asistido a talleres en la Escuela Internacional de Cine y Televisión de San Antonio de los Baños, Cuba, en su mayoría afiliados a CLACPI, o en otras escuelas de cine. La formación por lo general se realiza en talleres "express," es decir, muy cortos y comprimidos, adonde los participantes rotan en los roles de producción. Se capacita a hombres y mujeres, pero a la larga son más los hombres quienes continúan produciendo luego de los talleres. Se pone énfasis en el proceso colectivo de producción y con frecuencia se realizan consultas comunitarias para asegurar la conformidad de la comunidad con algún aspecto cultural. La formación suele centrarse en manejo de cámara y sonido, con poco tiempo para la instrucción dedicada a edición, teoría y crítica. Sin embargo, hay realizadores que han logrado cierta afinidad con alguna especialidad, y grupos que consiguen profundizar en determinadas técnicas como la fotografía, la animación stop-motion o digital, el guión de ficción, y la edición en programas de punta como Final Cut Pro. Cuando ciertas organizaciones logran este dominio, se vuelven los referentes regionales para otras comunidades y realizadores independientes produciendo video, asesorando distintas etapas de nuevas producciones, indígenas y no-indígenas. Es el caso de organizaciones como Ojo de Agua Comunicación en México, CEFREC-CAIB (Centro de Formación y Realización Cinematográfica, y Coordinadora Audiovisual Indígena-Originaria de Bolivia) en Bolivia, y Video nas Aldeias en Brasil, entre otras, quienes colaboran en un espíritu de reciprocidad no-comercial con otros productores de cine y video indígena'.

La mayor parte de la producción indígena de América Latina no aspira a replicar los convenios y normas del cine comercial. Esto puede deberse en parte a las circunstancias de producción, pero es más una elección consciente, una posición que rechaza un cine de consumo basado en la competencia tecnológica y de escaso contenido social. Ginsburg incluye tanto películas etnográficas e indígenas dentro de una categoría más amplia de los medios de comunicación etnográficos, acuñando el término "medios indígenas," como una forma de activismo cultural que "responde" a las estructuras de poder (Ginsburg, 1991). Juan Salazar (2004) propone la noción de "medios de comunicación imperfectos" ("imperfect media”) para refeerirse al video indígena, sobreponiendo la noción de García Espinosa del cine imperfecto a la rúbrica de Ginsburg ${ }^{10}$.

Las tradiciones de consulta comunitaria entran en juego cuando ocurren grabaciones en comunidades indígenas. En términos generales, los colectivos y no sólo los individuos inciden en la elección del tema del video. Las obras tienden a priorizar cuestiones de derechos humanos o territoriales, ya que los recursos suelen

\footnotetext{
9 En otras ocasiones he llamado este espíritu de cooperación una minga de los medios indígenas. 10 Estas nociones se discuten ampliamente en la tresis doctoral de Salazar (2004) y en Salazar y Córdova (2008).
} 
ser limitados y la mayoría de las comunidades indígenas están involucrados en algún modo de defensa de sus derechos. Las producciones son mayoritariamente en formato documental, seguido del docu-drama o docu-ficción, que ha sido usado muy exitosamente en la producción audiovisual de CEFREC-CAIB en Bolivia.

Al disponer de medios audiovisuales, las comunidades frecuentemente sienten la necesidad de contar su versión de la historia, para corregir la versión oficial de algún hecho o plantear un punto de vista tradicional en un conflicto que las afecta. Si bien la mayoría de las obras abordan los derechos, varias obras ofrecen estrategias de justicia o sanación tradicional para curar heridas históricas o coloniales. Tal es el caso de documentales como Somos alzados en nuestros bastones de mando (2006), que no sólo denuncia la represión de la policía colombiana en una pacífica recuperación de tierra, sino que explica cómo el pueblo nasa se defiende con sus bastones de autoridad tradicional, una vara tallada de madera. Armado sólo con sus bastones de mando, la Guardia Indígena, integrada por hombres y mujeres de la comunidad, acompaña las recuperaciones y marchas, de pie ante una policía militar fuertemente armada, en un contraste que es visualmente muy efectivo.

Muchas obras son el resultado de talleres de capacitación, por lo que pueden tener la sensación algo áspera de un ejercicio en lugar de una estética más "pulida." Esto revela el impulso democratizador de algunos de los procesos de formación audiovisual indígena, lo que Salazar ha llamado una "poética de los medios imperfectos" (Salazar, 2004). Las obras son producidos de forma colaborativa, dando lugar a formas híbridas, variados géneros e innovadoras metodologías que operan dentro de los valores tradicionales de reciprocidad. Como resultado, las producciones varían mucho en duración y tiempo de producción, lo que dificulta la postulación de proyectos para concursos y la planificación de estrenos de películas para festivales. Además, las obras pueden tener diferentes versiones para su distribución a los públicos internos y externos, y rara vez se producen con material promocional, fotografías en alta resolución, trailero página web.

Las ficciones, aunque escasas, a menudo se basan en cuentos tradicionales, involucran la memoria colectiva y pasan por consulta con los mayores para verificar la exactitud de la historia a tratar. Pueden recrear acontecimientos históricos importantes que han sido ignorados, borrados o mal representados por el imaginario nacional, generando un contrapunto. La voz o voces de estos documentales y ficciones suelen ser de la comunidad; son dialógicas y polifónicas. Las obras por lo general se hablan en lenguas indígenas, y con frecuencia son 
subtituladas en español o portugués para un público más amplio ${ }^{11}$. Este uso marcado de la lengua indígena refuerza la idea de resistencia y continuidad cultural.

\section{Más allá de indigenismo: la formación de videastas indígenas}

A finales de los años ochenta y hasta mediados de la década de los noventa, varios individuos y colectivos llevaron equipos y capacitación audiovisual a comunidades indígenas en diferentes partes de América Latina. Los primeros proyectos documentados fueron en Brasil y México en los años ochenta, seguidos por una notable iniciativa de capacitación a nivel nacional en Bolivia a mediados de los años noventa (el Plan Nacional Indígena Originario de Comunicación Audiovisual).

En México en 1989, el Instituto Nacional Indigenista (INI) creó un programa de formación audiovisual llamado Transferencia de Medios Audiovisuales a Comunidades y Organizaciones Indígenas. Patrocinados por el estado bajo una política de multiculturalidad, los Centros de Video Indígena (CVI) se establecieron en cuatro estados del país. Los CVI impulsan gran parte de la producción de video indígena en México, directa o indirectamente, aunque su influencia ha menguado, dando paso a colectivos de video indígena. En 1992, un grupo de realizadores formaron la Organización Mexicana de Videoastas Indígenas (OMVIAC), en un intento de crear una organización nacional independiente de los CVI, generando numerosas producciones y ayudando a realizadores emergentes a establecerse como comunicadores dedicados. Sin embargo, la organización se disolvió al año por falta de financiamiento y por la dispersión geográfica de sus miembros. La autoidentificación como "videoastas indígenas" de parte de los realizadores habla de la apropiación del término video indígena, y del deseo colectivo de unir fuerzas, a diferencia de otros directores independientes de la época. También se localiza al video indígena como una práctica distinta a otras formas de realización audiovisual.

Hoy existe un gran número de colectivos, realizadores independientes y festivales de video indígena en México ${ }^{12}$. En el estado de Michoacán se encuentra un fuerte movimiento de videastas purépechas, como Rául Máximo, Dante Cerano, Aureliano Soto y Pavel Rodríguez, además de proyectos colaborativos de formación audiovisual como el Centro de Producción Audiovisual de la Universidad Indígena y Campesina, que trabaja con comunidades nahuas en la costa. En el vecino estado

\footnotetext{
11 Una fracción de la obra se traduce con subtítulos en inglés o francés, con la excepción de las obras de Vídeo nas Aldeias, que son regularmente subtitulados en inglés, francés, portugués y español, y Promedios, basada en San Cristóbal y Chicago, subtitula al inglés y distribuye directamente sus producciones a festivales, instituciones académicas y al público en general. 12 Para un listado más amplio de las organizaciones de medios indígenas en México, ver Córdova y Zamorano (2004), y también Ramos y Castells-Talens (2010), quienes discuten el rol del estado y las radios comunitarias en el desarrollo del video indígena en México.
} 
de Guerrero trabajan Altepetl Nahuas de la Montaña de Guerrero, la organización independiente Ojo de Tigre Comunicación/Mirada India, liderada por el realizador nahua José Luis Matías, y el Centro de Derechos Humanos de la Montaña Tlachinolan.

En el sur del país trabajan organizaciones y realizadores en los estados de Oaxaca, Chiapas y Yucatán. En Oaxaca, Ojo de Agua Comunicación capacita y produce videos y radio, prestando equipo y asesorías a realizadores independientes de la región. Otras organizaciones realizan producción y teledifusión regional: TV Tamix y Comunalidad llevan más de diez años produciendo y transmitiendo programas en Mixe y Zapoteco en las comunidades de la Sierra Norte. Entre las organizaciones de base que usan el video con sus comunidades o en sus regiones están el Grupo Solidario de Quiatoni en la región zapoteca de la Sierra Sur, el Centro por los Derechos de la Mujer Nääxwiin, la Casa de la Mujer Rosario Castellanos y la Unión de Comunidades Indígenas de la Zona Norte del Istmo, en la región del Istmo Tehuantepec.

Los festivales de cine indígena han facilitado el encuentro con el público en general y han creado un espacio crítico para los realizadores indígenas de México. El Festival Internacional de Cine de Morelia (en Michoacán) desde su segunda edición en 2002 y hasta 2010 realizó un Foro Indígena como parte regular del festival, con mesas de discusión y muestras especiales. Desde 2005 se realiza en la misma ciudad el Festival de Video Indígena en Morelia, organizado por el Centro de Video Indígena del estado, adonde se presentan y premian obras de realizadores indígenas de todo el país. En Oaxaca se organizan muestras en espacios públicos como el Zócalo y en la sala gratuita del Cineclub el Pochote, y en 2006 se realizó el VIII Festival Internacional de Cine y Video de los Pueblos Indígenas, un festival latinoamericano rotativo de alcance internacional organizado por CLACPI.

A través del video, los trabajadores indígenas migrantes y sus organizaciones se mantienen en contacto con sus comunidades de origen, e intercambian sus experiencias como migrantes. El Frente Oaxaqueño Indígena de Organizaciones Binacionales (FIOB) trabaja en video y radio, mantiene un sitio web y un boletín para fortalecer su labor de concientización sobre la situación y los derechos de los migrantes. También hay algunos directores indígenas que han migrado que continúan su labor creativa desde Estados Unidos o Europa, como el director mixe Carlos Efraín Pérez, quien ha fundado su propia productora, Mecapal Films, en Lyon, Francia.

En 1994 la atención internacional se dirigió a las comunidades mayas de la región cuando éstas se levantaron por la defensa de sus tierras y autonomía con el Ejército Zapatista de Liberación Nacional. Varias de las actuales comunidades autónomas zapatistas actualmente usan el video para mandar comunicados al 
exterior, e internamente como herramienta para definir y promover experiencias comunitarias desde la resistencia. Desde 1998, la organización binacional Promedios de Comunicación Comunitaria (Chiapas Media Project en Estados Unidos) aportó equipos y capacitación en video a estas comunidades.

Otro grupo de medios indígenas en Chiapas es el Proyecto Videastas Indígenas de la Frontera Sur que, trabajando desde el Centro de Investigaciones y Estudios Superiores en Antropología Social y el Centro de Estudios Superiores de México y Centroamérica, Universidad de Ciencias y Artes de Chiapas (CESMECAUNICACH). Este proyecto organiza talleres y diplomados para jóvenes indígenas y programa foros de video indígena a los que asisten participantes de todo el país. En San Cristóbal de las Casas, el centro cultural Sna Jtz'ibajom se ha aliado con grupos de producción independiente para hacer talleres de video en lenguas autóctonas de la región. En la zona de Palenque, el Comité de Defensa de la Libertad Indígena Xi'nich produce videos sobre la lucha de los indígenas y sus tradiciones, las cuales han circulado a nivel internacional.

En Yucatán la organización Yoochel Kaaj organiza talleres de video para jóvenes de comunidades mayas desde 1998. Turix es una video-revista producida en talleres de capacitación por jóvenes realizadores maya, zapoteco, mixteca, tzeltal y chol. Estos trabajos circulan en las comunidades donde fueron filmados $y$ en comunidades aledañas. Yoochel Kaaj también organiza un festival regional anual llamado Geografías Suaves, con iniciativas que alcanzan regiones fronterizas con Guatemala y Belice. En el año 2000 entró en operación en Yucatán el más nuevo de los cuatro Centros de Video Indígena, el cual empezó a trabajar en conjunto con organizaciones locales dictando talleres, ofreciendo servicios de producción y desde 2011, su propio festival, el Festival Kayché Tejidos Visuales, abocado a temas de migración, soberanía alimentaria, autogestión y saberes indígenas.

\section{Visiones amazónicas de las aldeas para el mundo}

El proyecto más conocido en sudamérica fue el Proyecto de Video Kayapo, fundada en 1990 por el antropólogo Terrence Turner quien capacitó a comuneros kayapó para filmar y editar videos promocionales. Turner había trabajado con los kayapo desde 1962, realizando películas etnográficas con la British Broadcasting Company y Granada Television International. Mientras que las películas kayapó son bien conocidos por el gran impacto político que tuvieron en su momento, los kayapó no continuaron trabajando con video para circulación externa.

El trabajo dedicado de la organización brasileña de Video nas Aldeias (VNA) comenzó con el fotógrafo y activista Vincent Carelli, cuyas primeras experiencias de vida con las comunidades Xikrin en la Amazonía lo llevó a una vida de promoción en favor de los pueblos indígenas. Después de colaborar con la Fundación Nacional 
del Indio (Fundação Nacional do Indio - FUNAI), Carelli ayudó a fundar el Centro de Trabalho Indigenista (CTI) y trabajó en un banco de imágenes para el Centro Ecuménico de Documentación e Información. A partir de estos dos proyectos surgió la idea de Vídeo nas Aldeias, que comenzó a tomar forma en 1985. El uso de la palabra "video" en el nombre del proyecto indica más que una opción técnica, convirtiéndose en una metodología de esta iniciativa, única en su tiempo, que utilizaba el sitio de la aldea como el lugar de la acción (nas Aldeias es "en los pueblos" en portugués), así dejando de lado la estigmatización y las expectativas de pureza que puede conllevar términos como "indio"o "indígena."

El primer taller de VNA se inició en 1987, y consistió en la toma de videos grabados en una aldea para ser visto por otro grupo de indígenas en la región. Entre 1983 y 1998, Carelli colaboró con la antropólogas Virginia Valadão y Dominique Gallois, cambiando gradualmente el concepto del proyecto desde la simple documentación de la vida indígena, a la formación de los indígenas para registrar sus propias vidas. Se presentó el nuevo reto de formar a nuevos realizadores, y en 1998 la directora de cine y editora Mari Corrêa se incorporó al proceso, trabajando con Carelli para establecer formalmente Vídeo nas Aldeias como una organización independiente, no gubernamental en el año 2000. El realizador ashaninka, Isaac Pinhanta describe este nuevo enfoque:

Estamos utilizando el instrumento de video de una manera diferente, a nuestra manera. Lo usamos también para ayudar a entender mejor la sociedad, en la forma en que pensamos, nosotros, aquí y allí. Así es como somos, tenemos nuestro propio conocimiento y sería bueno si todo el mundo empezara a ver esto de ahora en adelante ... Podemos organizar con el mismo instrumento que utilizan los blancos, pero con una imagen y propósito diferente. Que lo utilice de acuerdo a sus necesidades y su forma de organizar a ti mismo (Pinhanta, 2004:17).

Video nas Aldeias ha trabajando incansablemente desde entonces con más de cuarenta comunidades indígenas del Brasil y en las fronteras del país con Perú y Paraguay, para producir videos documentales sobre la base de resistencia de prácticas culturales y vida comunitaria. Los cineasta de VNA participan en talleres de capacitación donde estudian tanto el manejo de cámara como técnicas de edición, habilidades que pueden perfeccionar en estaciones de edición en sus propias aldeas. VNA trabaja con un equipo interdisciplinario de entrenadores que colaboran con los líderes indígenas para formar la próxima generación de directores indígenas, a menudo trabajando in situ en la comunidad. Hasta la fecha, VNA ha producido más de 70 videos, la mitad de los cuales son de autores indígenas. Sus logros incluyen la creación de un programa de televisión indígena, una biblioteca y un sistema de circulación de vídeo para los pueblos indígenas, la facilitación de 
talleres nacionales y regionales para el intercambio de realizadores, y la organización de una reunión nacional anual de cineastas indígenas.

Además, las producciones de VNA han sido exhibidas en festivales a lo largo de América y en Europa, ganando numerosos premios. El trabajo está siendo distribuido en colecciones de DVD, y suben clips a su portal bilingüe (www.videonasaldeias.org.br, en inglés y portugués). Los videos son hablados en sus lenguas originarias y la mayoría también cuentan con subtítulos en portugués, inglés, francés, italiano y español. VNA ha ganado el continuo apoyo de la Agencia Noruega de Cooperación para el Desarrollo, una oficina bajo la directiva del ministerio noruego de Asuntos Exteriores. En 2005, VNA fue reconocido por el Ministerio de Cultura de Brasil como un Pontao de Cultura, (mega-punto de cultura), un centro cultural de la región dentro de una red de centros culturales que ya operan con éxito en diversas regiones de Brasil.

Aunque podría decirse que cualquier proyecto de video indígena es un proyecto político, VNA se ha destacado entre sus organizaciones pares como una iniciativa de formación y difusión de alto nivel de producción, proporcionando no sólo la formación y el acceso a los equipos de grabación de vídeo, sino además el entrenamiento de edición y facilitación de equipos, así formando una generación de realizadores indígenas diversamente hábil, visualmente entrenados y con conocimientos tecnológicos de punta. Por lo tanto, Video en las Aldeias, como señala su nombre, habla de un enfoque y una metodología de capacitación y trabajo, no sólo del cuerpo de obra resultante.

\section{Organizaciones nacionales y redes regionales}

En Colombia y Ecuador, los colectivos de comunicación al interior de las organizaciones indígenas regionales y nacionales comenzaron a producir video más regularmente. El uso del video en el contexto es parte del proceso organizativo; responde a una necesidad de divulgar actividades dentro de la comunidad, de crear herramientas de capacitación y diseminación al exterior, y es usado como mecanismo de denuncia ante la violencia. Se aborda la defensa de los derechos humanos, de la mujer y del territorio, se documentan fiestas, medicina tradicional, y diversos aspectos de continuidad cultural, junto a las luchas tanto del presente como del pasado del movimiento indígena. También se trata el tema del desplazamiento, tanto de la migración del campo a la ciudad como el desplazamiento por desastres naturales o violencia militar, y se ha abordado el impacto de grandes megaproyectos de "desarrollo" de las trasnacionales o de los tratados de libre comercio.

En Colombia, los organismos indígenas ACIN, CRIC y ONIC producen video desde los años noventa, principalmente documentales. Estas organizaciones han creado sus Departamentos de Comunicación, como el de ACIN, que cuenta con el 
Tejido de Comunicación, que impulsa desde 2010 la Escuela de Comunicación, un ciclo de talleres de capacitación en radio y video para jóvenes en la zona. El limitado acceso a las herramientas de producción lleva a que algunas organizaciones opten por contratar o autorizar a realizadores no-indígenas comprometidos con la causa para que graben sus eventos o documentales. Es el caso de Fundación Cine Documental (los cineastas Jorge Silva y Marta Rodríguez), que mediante acuerdos con las organizaciones y comunidades indígenas de Colombia documentan diversas actividades de la comunidad, enfocado a los derechos humanos, y que están entre las primeras personas en registrar recuperaciones de los territorios indígenas y aspectos culturales de los pueblos. Su largometraje documental Nuestra Voz de Tierra Memoria y Futuro (1974 - 1980) (1982), de corte experimental, ganó el Premio a la Mejor Realización Latinoamericana en el Primer Festival de Cine de los Pueblos Indígenas (México), el festival fundacional de CLACPI, organización adonde la cineasta Marta Rodríguez aún participa activamente.

Existen diversos proyectos colaborativos en Colombia, como el colectivo Zhigoneshi (Sierra Nevada de Santa Marta, en el norte de Colombia), en que participan comunicadores arahuacos, kogi y wiwa junto al documentalista Pablo Mora; proyectos de investigación académica que buscan participación de la comunidad como el Shamans' Videohistory Project, en Colombia, iniciado 2003 por el chileno Carlos Salinas; y existen co-producciones internacionales, como el documental Dueños del Agua (2009), dirigida colectivamente por la antropóloga estadounidense Laura Graham, el director xavante Caime Waiasse de Brasil, y el director wayuu David Hernández Palmar, y que es distribuida por Documentary Educational Resources, en Estados Unidos.

La circulación de estos trabajos continúa siendo bastante limitada. Se realizan muestras comunitarias con cierta frecuencia, pero son esporádicas y no cuentan con financiamiento estable. Los líderes políticos hacen uso del video para fines educativos y para sus giras por diversas regiones y países. Son los festivales internacionales los que les dan mayor visibilidad a los trabajos. Colombia tiene desde 2010 dos festivales de video indígena anuales y estables: Daupará, que se ha realizado en Bogotá y Popayán, y el festival de Cine y Video indígena Rodolfo Maya, en Cauca. Algunos de los documentales de ACIN, se suben a Internet mediante su página web (www.nasacin.org). ACIN ha logrado distribución internacional de uno de sus trabajos, Somos Alzados en Nuestros Bastones de Mando (2006), distribuido por Third World Newsreel (www.twn.org), una agencia basada en Nueva York. El contacto con esta distribuidora se logró mediante la exhibición de este video en el 2006 Native American Film + Video Festival (XII Festival de Cine + Video Indígena Americano realizado en Nueva York en 2006), organizado por el Museo Nacional del Indígena Americano de la Institución Smithsonian 
(www.redesindigenas.si.edu). En Venezuela, la primera Muestra de Cine Indígena de Venezuela realizada en 2008 se ha convertido el en Festival Internacional de los Pueblos Indígenas (FIPI), apoyado por la Fundación Cinemateca Nacional, la Fundación Villa del Cine, y el Ministerio del Poder Popular para la Cultura, demostrando un avance en políticas comunicacionales hacia las comunidades indígenas.

En los años noventa, surgió en Ecuador un gran pionero del video indígena, el realizador kichwa Alberto Muenala, quien realiza documentales y ficciones muy innovadores desde 1992 que se han exhibido mundialmente, siendo reconocidos por el cine experimental ecuatoriano (Alvear y León, 2009). Muenala también publicó textos críticos, siendo el primer cineasta indígena que además teoriza en torno a su producción y sobre el video indígena en foros internacionales (Muenala, 1995) y participó con CLACPI y la CONAIE en la organización de festivales y en formación de numerosos realizadores indígenas en Bolivia, Ecuador y México. Muenala ha sido de los pocos proponentes de la ficción, y realizó talleres de capacitación adonde la ficción era el principal vehículo narrativo para contar historias comunitarias. Una serie de ficciones indígenas del taller dictado en Bolivia han sido exhibidos y premiados en diversos festivales.

La producción audiovisual de la CONAIE se aboca al fortalecimiento de lenguas y culturas indígenas, y ha organizado dos festivales internacionales de video indígena en Ecuador: el I Festival Continental de Cine y Video de las Naciones de Abya-Yala en 1994 y el X Festival Internacional de Cine y Video de los Pueblos Indígenas en octubre de 2010. En Ecuador trabajan además colectivos como la Corporación de Productores Audiovisuales de las Nacionalidades y Pueblos (CORPANP), que capacita y produce documentales con jóvenes indígenas en colaboración con el realizador kichwa Franklin Quizpe de la comunidad de Saraguro. La CORPANP realiza talleres de video y muestras como la Muestra Itinerante de Cine Documental de las Nacionalidades y Pueblos de Ecuador Kikinyari, que han llegado a ser presentada incluso en el Perú. Tanto CONAIE como CORPANP hoy participan de CLACPI.

En la amazonía ecuatoriana se realizan diversos trabajos audiovisuales al interior de las comunidades, como la premiada obra del fotógrafo, músico y documentalista kichwa Eriberto Gualinga, de la comunidad de Sarayaku. Sus obras Sachata Kishipichik Mani/Soy Defensor de la Selva (2003) y Sisa Nambi/El Camino de las Flores (2010) han ganado premios en festivales internacionales como el festival de CLACPI en 2006 y 2010 respecivamente. El trabajo de Gualinga demuestra un cuidadoso manejo de la cámara y del sonido, incorporando el canto de mujeres kichwas y sonido ambiental de la selva en su banda sonora. Su estética es de observación pausada del entorno natural, adonde la selva no es un fondo sino un 
protagonista de la obra. Al mismo tiempo, sus obras son registros de resistencia indígena, usadas ampliamente en la difusión de la defensa territorial de la comunidad de Sarayaku.

En Bolivia, el Sistema Nacional Indígena Originario de Comunicación Audiovisual (conocido inicialmente como Plan Nacional de Comunicación Indígena, o simplemente "el Plan") fue lanzado en 1996, junto con la Coordinadora Audiovisual Indígena-Originaria de Bolivia (CAIB). Esta amplia iniciativa de formación y producción de medios de comunicación a nivel nacional destinada a apoyar la auto-representación indígena, apuntaba a la formación de indígenas originarios de diversas naciones, federaciones campesinas, organizaciones regionales y las comunidades de migrantes en la producción de video. El Plan es coordinado conjuntamente por el Centro de Formación y Realización Cinematográfica (CEFREC) y CAIB, que incluye tres de las principales organizaciones y confederaciones indígenas de Bolivia ${ }^{13}$. Esta parcería ha generado más de 200 producciones entre documentales para transmisión en televisión comunitaria, ficciones basadas en cuentos tradicionales, reportajes, documentales, $y$ docu-dramas, ganando premios en festivales internacionales en Latinoamérica, Europa y Norteamérica. El trabajo de CEFREC y CAIB se ha extendido hasta el resto del Cono Sur, realizando talleres de formación de video en Chile y Paraguay, e invitando a líderes del noroeste argentino y del norte chileno a participar en capacitaciones y encuentros. CEFREC y CAIB han sido instrumentales en la capacitación de miembros de CLACPI, y en asegurar el financiamiento y la continuidad del CLACPI y sus festivales.

Otras regiones de América Latina cuentan con organizaciones indígenas nacionales (Argentina, Brasil, Chile, Paraguay, Perú, Suriname y Venezuela), pero la mayoría de ellas carecen de fondos suficientes para trabajar regularmente con el audiovisual. El caso más llamativo es el de Perú, que cuenta con una vasta población indígena, una fuerte red de defensa de derechos indígenas, una vasta historia del cine etnográfico, sin embargo, muy poca producción audiovisual indígena. Una serie de iniciativas independientes trabajan en medios de comunicación con pueblos indígenas en Argentina, Chile, El Salvador, Guatemala, Nicaragua, Panamá, Paraguay, Perú, Suriname y Venezuela, pero este trabajo no recibe distribución formal.

Argentina y Chile carecen de políticas nacionales de formación de audiovisual indígena. Algunos antropólogos, periodistas, cineastas y agrupaciones activistas han trabajado junto a comunidades indígenas para filmar documentales, pero pocos

13 El trabajo de CEFREC y CAIB se encuentra ampliamente documentado por Schiwy (2002, 2009), Himpele $(2004,2008)$ y por la antropóloga mexicana Gabriela Zamorano, quien presentó su estudio como tesis doctoral en 2009. 
de estos proyectos de colaboración se han tornado permanentes o sostenibles. En cierto sentido, esto puede haber favorecido el desarrollo de un video indígena más autónomo en la región. Es desde este territorio que surge una de las realizadores indígenas más notables y una figura clave en la organización de CLACPI, la realizadora mapuche Jeanette Paillán.

En 1997, Endesa, la mayor empresa eléctrica de América Latina, comenzó a construir un gran complejo hidroeléctrico denominado Ralco, en el curso superior del río Bío Bío, en territorio pehuenche. La resistencia pehuenche levantó un amplio frente de oposición a la presa. Muchos miembros de la comunidad fueron desplazados con el tiempo y en los dos años previos a la construcción, la periodista Jeanette Paillán grabó Punalka: El Alto Bío Bío (1995), una visión curiosamente poética dentro de la lucha por la protección del río amenazado. Paillán produjo la película a través de su propia compañía de producción Lulul Mawida. El texto de la película es un poema en mapuzungun del poeta mapuche Leonel Lienlaf, y es más sobre el espíritu del río que una cronología de una lucha. El video fue grabado en Betacam y se proyectó en 1997 Native American Film + Video Festival.

Paillán se convirtió en la primera realizadora mapuche reconocida internacionalmente, una hazaña notable para una mujer indígena sin formación cinematográfica formal. Sin embargo, esto no la protegió de sufrir golpes y de que le arrebataran su cámara mientras cubría protestas. Más adelante viajó a Cuba, México, Europa y África del Sur para denunciar las violaciones de los derechos humanos de su pueblo y el saqueo de sus tierras. Paillán continúa haciendo videos, entre ellas Wallmapu (2003), un recuento histórico del despojo del territorio mapuche desde el punto de vista mapuche, que obtuvo una Mención Especial del Jurado a la Mejor Investigación Histórica en el Festival DerHumALC 2003 (Quinta Festival Internacional de los Derechos Humanos) en Santiago del Estero, Argentina. En 2008 recibió una beca de cine en España y produjo su primer corto de ficción, Perimontún (2008).

Paillán además ha ganado importancia en CLACPI, y en la formación de una red de comunicadoras indígenas a nivel continental. En 2004, organizó el VI Festival Internacional de Cine y Video de los Pueblos Indígenas en Santiago, que incluyó un taller de formación audiovisual para jóvenes indígenas. En 2008 fue honrada en España por su defensa de la cultura de los pueblos indígenas y el patrimonio con el Premio Ciudad de Córdova a la Comunicación de la Solidaridad. En 2010 fue reelecta como Coordinadora General de CLACPI.

En 2004, el director chileno Juan Francisco Salazar dirigió un documental sobre los medios de comunicación mapuche, De la tierra a la pantalla (2004), que conjuga el activismo de tres diferentes proyectos de medios de comunicación mapuche: Jvken Mapu, el más antiguo programa de radio mapuche y bilingue en Chile; Lulul 
Mawida, dirigido por Jeanette Paillán, y Mapuexpress, una agencia de noticias electrónica mapuche. Los tres colectivos son auto-gestados, trabajando en medios de comunicación hace mucho tiempo. El año siguiente el realizador Anthony Rauld produjo un corto sobre Jvken Mapu, llamado Wixage Anai (2005), que recibe una mención especial en el octavo festival CLACPI de 2006. En 2009, los documentalistas argentinos Emilio Cartoy Díaz y Cristián Jure lanzan La Guerra Por Otros Medios (2010), sobre cómo las comunidades indígenas en Argentina, Bolivia y Brasil se están empoderando mediante los medios de comunicación. El surgimiento de documentales sobre los procesos de apropiación de los medios por parte de comunicadores indígenas como fenómeno social y no como experimento antropológico indica la inserción definitiva de una línea de trabajo audiovisual y cultural que comienza a tener resonancia incluso en el alejado mundo del cine.

\section{Conclusiones: algunos aportes del video indígena}

Mediante la producción de obras experimentales, narrativas y documentales, activistas culturales indígenas han adaptado tecnologías de nuevos medios de comunicación para documentar historias subalternas, preservar el territorio de los proyectos multinacionales y fortalecer la identidad tradicional y contemporánea. El video, en particular, se ha convertido en una poderosa herramienta para difundir la situación de los pueblos indígenas a escala local y global, así como un medio de expresión creativa y de continuidad cultural. Estas formas específicas de cine y video producido por los pueblos indígenas de todo el mundo se conocen como medios de comunicación indígenas, y en América Latina, como "video indígena."

No existe un único hilo unificador que sostenga las obras de video indígena. En gran medida se mantienen unidos por un enfoque similar en el hacer del trabajo audiovisual, realizado desde una perspectiva conjunta, comunitaria e indígena, y que se apoya en vínculos transnacionales de activistas, cineastas, periodistas, académicos, fondos y programadores que se conectan a una comunidad dispersa de los productores de video indígena y ayudan a circular su obra. Ciertas iniciativas desarrolladas a finales de los años ochenta y hasta mediados de la década de los noventa llevaron la capacitación en video y el equipamiento básico a comunidades indígenas en diferentes partes de América Latina para fomentar su comunicación propia. Los primeros proyectos documentados se realizaron en Brasil y México, seguidos por un sistema de capacitación nacional en Bolivia.

Varias organizaciones y personas intervienen y colaboran en los distintos niveles alrededor de video indígena, para estimular la formación de los pueblos indígenas en la tecnología de vídeo y apoyar la difusión de su trabajo. Entre ellas se incluyen proyectos académicos, productores independientes, organizaciones regionales, proyectos nacionales y estatales, organizaciones no gubernamentales y centros de 
formación indígena, organismos internacionales de apoyo, archivos fílmicos y festivales, y cada vez más, programas y canales de televisión indígena y comunitarios.

Una notable innovación del video indígena es el uso de la lengua indígena dentro de las producciones, lo que las hace accesibles a miembros de la comunidad, y a la vez recalca la importancia del uso de la lengua para los jóvenes, quienes están más expuestos a perderla. Otro gran aporte es la puesta en escena de cuentos tradiciones contadas tradicionalmente mediante la oralidad, grabadas como cortos de ficción. Hace poco se ha visto un mayor número de largometrajes indígenas en Latinoamérica, como el documental La Pequeña Semilla en el Asfalto (2010, 70 min., México), del realizador tsotsil Pedro Daniel López, que fue terminado en cine gracias a fondos del Instituto Mexicano de Cinematografía. Esta obra documenta la experiencia de jóvenes tsotsiles que migran de sus comunidades a la ciudad de San Cristóbal en busca de oportunidades de formación y desarrollo creativo. El realizador purépecha Dante Cerano dirigió el primer largo de ficción indígena en latinoamérica, Uaricha/ En la Muerte (2004, 68 min., México), y CEFREC-CAIB ha realizado una docu-ficción de largometraje en la amazonía boliviana, El Grito de la Selva (2008, 95 min., Bolivia).

Tal vez el cambio más notorio que ha traído el video indígena es la visibilización de realidades indígenas contemporáneas narradas en voz propia, sin la habitual distorsión de los medios masivos de comunicación. O sea, una ruptura de la invisibilidad y exclusión social, el desplazamiento del estereotipo del indígena como marginal, y la proyección de una diversidad cultural, sabiduría, planes de vida y vigencia de los pueblos indígenas. Se ha dado mediante la comunicación indígena una mayor posibilidad de acceder a mejor información sobre la realidad indígena; según reflexiona un antiguo dirigente del CRIC,

de alguna manera los indígenas han entendido que pueden buscar los mecanismos de hacer difusión de su situación sin esperar que de afuera se haga, creándose cierta conciencia de la necesidad del manejo de estos medios como instrumentos de lucha dentro de la defensa de sus derechos. Además nos ha hecho tomar conciencia de que los medios de comunicación son importantes; pueden destruir o apoyar, según las manos en que se encuentren (Jesús Avirama, comunicación personal, octubre 2011).

Un espacio importante en la articulación de este verdadero movimiento es el festival de cine indígena, donde los medios de comunicación indígenas llegan a la comunidad, a públicos regionales e internacionales, y adonde los realizadores se reúnen a debatir con sus compañeros y colaboradores. Actualmente existen más de sesenta y cinco festivales de cine especializados en el cine y video de los pueblos indígenas, de los cuales casi la mitad se realizan en Estados Unidos y seis en Canadá (Córdova, 2012). Gracias a estas reuniones, el audiovisual indígena se ha ganado un 
lugar cada vez más importante no sólo dentro de las culturas locales de cine de América Latina y dentro de sus comunidades, sino también en los ámbitos de cine nacional, documental, de derechos humanos y mundial.

\section{Referencias}

Acosta, M., dir. (2006). Somos Alzados en Nuestros Bastones de Mando. DVD. Colombia: ACIN.

Alvear M., y León, C. (2009). Ecuador Bajo Tierra: videografías en circulación paralela. Quito: Ochoymedio.

Benamou, C. (1994). Entrevista a Jesús Avirama (video). Smithsonian National Museum of the American Indian. Transcripción de la autora.

Cartoy Díaz, E. y Jure, C., dir. (2010). La Guerra por Otros Medios. DVD. Argentina: Masato Media.

CEFREC- CAIB, dir. [dirección colectiva]. (2010) Sirionó. DVD. Bolivia: CEFREC- CAIB.

Cerano, D., dir. (2004). Uaricha En la Muerte. VHS. México: Exe Video.

Córdova, A. y Zamorano, G. (2004). "Mapeando Medios en México: Video Indígena y Comunitario en México," Native Networks/Redes Indígenas, Smithsonian National Museum of the American Indian, Oct. 2005. Obtenido el 11 de noviembre de 2011 en: http:/ / www.nativenetworks.si.edu/esp/rose/mexico.htm\#open.

Córdova, A. (2006). "El Largo Camino Hacia la Comunicación Propia." Entrevista a Mariano Estrada. Native Networks/Redes Indígenas, Smithsonian National Museum of the American Indian, Mayo 2006. Obtenido el 12 de diciembre de 2011: http://www.nativenetworks.si.edu/esp/rose/estrada_m_interview.htm.

- (2012) “Towards an Indigenous Film Festival Circuit," En Film Festival Yearbook 4: Film Festivals and Activism. Glasgow: University of St. Andrews.

Corrêa, M. y Carelli, V., dir. (2008) De Volta a Terra Boa. DVD. Brasil: Video nas Aldeias.

Corrêa, M., Ikpeng, K., y Txicão, K., dir. (2007). Pirinop: Meu Primeiro Contato. DVD. Brasil: Video nas Aldeias.

Declaración de la Cumbre Continental de Comunicación Indígena de Abya Yala (2010). Cumbre Continental de Comunicación Indígena de Abya Yala, Noviembre 2010. Obtenido el 11 de noviembre de 2011 en http://www.cccia-2010.com/.

Frota, M. (1993). “Taking Aim e a Aldeia Global: a Apropriação Cultural e Política da Tecnologia de Vídeo pelos Índios Kayapós,” Mnemocine: Memoria e Imagem. Obtenido el 5 de mayo de 2011 en http://www.mnemocine.com.br/osbrasisindigenas/frota.htm.

García Espinosa, J. (1979 [1969]). “For an imperfect cinema.” (Traducción Julianne Burton) Jump Cut: A Review of Contemporary Media, No 20, pp. 24-26. 
García Hurtado, F., dir. (1984). Tupac Amaru. 35mm. Cuba/Perú: Cinematográfica Kuntur e Instituto Cubano del Arte e Industria Cinematográfica.

Getino, O. y Solanas, F. E. (1969). "Hacia un tercer cine: Apuntes y experiencias para el desarrollo de un cine de liberación en el Tercer Mundo." Hojas de cine: testimonios y documentos del Nuevo Cine Latinoamericano: Volumen I. Obtenido el 3 de marzo de 2012 en http://www.cinelatinoamericano.cult.cu/biblioteca/ fondo.aspx? $\operatorname{cod}=2408$.

Ginsburg, F., Abu-Lughod, L. y Larkin, B. (2002). “Introduction.” En Media Worlds: Anthropology on New Terrain. Los Angeles: University of California Press. pp 1-31.

Ginsburg, F. (1991). “Indigenous Media: Faustian Contract or Global Village?” En Cultural Anthropology No 6 (1).pp. 92-112.

(1994). "Embedded Aesthetics: Creating a Discursive Space for Indigenous Media." Cultural Anthropology No 9 (3).pp 365-82.

Graham, L. Waiasse, C. y Hernández Palmar, D., dir. (2009). Ö Tede'wa/Dueños del Agua. DVD. Brasil/Estados Unidos: Associação Xavante de Pimentel Barbosa/DER.

Gualinga, E., dir. (2003). Sachata Kishipichik Mani. DVD. Ecuador: Selvas Producciones.

-------- (2010). Sisa Nambi. DVD. Ecuador: Selvas Producciones.

Halkin, A. (2008). "Outside the Indigenous lens: Zapatista and Autonomous Videomaking.” En Global Indigenous media: Cultures, Poetics and Politics. Durham: Duke. pp. 160-180.

Himpele, J. (2004, junio). "Packaging Indigenous Media: An Interview with Ivan Sajinés and Jesús Tapia.” American Anthropologist. No 2, Vol. 106. pp 354-362.

(2008). Circuits of Culture: Media, Politics and Indigenous Identity in the Andes. Minneapolis, London: University of Minnesota Press.

Leyva, X. y Köhler, A. (2004). Proyecto Videoastas Indígenas de la Frontera Sur. Centro de Investigaciones y Estudios Superiores en Antropología Social, Unidad Sureste. Obtenido el 16 de marzo de 2012 en http://sureste.ciesas.edu.mx/Investigacion/Proyectos\%20especiales/Proyectos/P VIFS/espanol/pvifs.html.

López, P. D., dir. (2010). La Pequeña Semilla en el Asfalto. DVD. México: Mundos Inéditos e IMCINE.

Magallanes-Blanco, C. (2008). The Use of Video for Political Consciousness-Raising in Mexico: An Analysis of Independent Videos About the Zapatistas. Lewiston, Queenston, Lampeter: Edwin Mellen Press.

Muenala, A. (1995). "Cinema as an instrument for indigenous peoples identity," Felix: $A$ Journal of Media Arts, No 1, Vol. 2.pp. 154-156. 
Noza, A., Ipamo, N., y Sanjinés, I., dir. (2008). El Grito de la Selva. DVD. Bolivia: CEFRECCAIB.

Paillán, J., dir. (1995). Punalka: El Alto Bío Bío. VHS/U-matic. Chile: Lulul Mawida.

---- (2003). Wallmapu. DVD. Chile: Lulul Mawida.

--------- (2008). Perimontún. DVD. España: Lulul Mawida.

Pinhata, I. (2004). "Voçe vê o mundo do outro e olha para o seu." En Mostra Vídeo nas Aldeias: Um Olhar Indigena. Rio de Janeiro: Banco do Brasil. pp 12-20.

Rauld, A., dir. (2005). Wixage Anai: Despierta y Levanta. DVD. Chile: Jvken Mapu.

Salazar, J. F. (2004). Imperfect Media: The Poetics of Indigenous Media in Chile. Tesis doctoral en comunicación y medios. Dir: H. Cohen. University of Western Sydney.

------- (2004). De la Tierra a la Pantalla. DVD. Australia/Chile: Moai Films.

Salazar, J. F., y Córdova, A. (2008). "Imperfect Media and the Poetics of Indigenous Video in Latin America." En Global Indigenous Media: Cultures, Practices and Politics. Durham: Duke.pp. 39-57.

Schiwy, F. (2002). "La Otra Mirada: Video Indígena y Descolonización.” En Indisciplinar las Ciencias Sociales: Geopolíticas del Conocimiento y Colonialidad del Poder, Perspectivas desde lo Andino. Quito: Ediciones Abya Yala.

(2003). “Decolonizing the Frame: Indigenous Video in the Andes.” Framework Vol. 44 (1).pp. 116-132.

(2009). Indianizing Film: Decolonization, The Andes, and the Question of Technology. New Brunswick: Rutgers.

Shohat E., y Stam, R. (1994). Unthinking Eurocentrism: Multiculturalism and the Media. New York: Routledge. 1994.

Silva, J. y Marta Rodríguez, M., dir. (1982). Nuestra Voz de Tierra Memoria y Futuro (1974 - 1980).VHS. Colombia: Fundación Cine Documental.

Turner, T. (1990). "Visual Media Cultural Politics and Anthropological Practice: Some Implications of Recent Uses of Film and Video among the Kayapo of Brazil." CVA Review (Commission on Visual Anthropology), Spring).pp 8-13.

(1991). "The Social Dynamics and Personal Politics of Video Making in an Indigenous Community." Visual Anthropology Review 7 (2).pp 68-76.

(1995 [1992])."Representing, Resisting, Rethinking: Historical Transformations of Kayapo Culture and Consciousness.” En Colonial Situations: Essays on the Contextualization of Ethnographic Knowledge. Madison: University of Wisconsin. pp. 285-313. 
(2002). "Representations, Politics, and Cultural Imagination in Indigenous Video: General Points and Kayapo Examples." En Media Worlds: Anthropology on New Terrain. Berkeley: University of California. pp 75-89.

United Nations Declaration on the Rights of Indigenous Peoples. (2006). Office of the United Nations High Commissioner for Human Rights. Obtenido el 16 de marzo de 2011 en http://www2.ohchr.org/english/issues/indigenous/declaration.htm

Wortham, E. (2004, junio). "Between State and Indigenous Autonomy: Unpacking Video Indígena in Mexico." American Anthropologist. No 2, Vol. 106. pp. 363-367.

Yube, Z., dir. (2006). Xina Bena. DVD. Brasil: Video nas Aldeias.

------- (2008). Ja me transformei em Imagem. DVD. Brasil: Video nas Aldeias.

Zamorano, G. (2005). “Community Video and Self-representation.” Entrevista a Carlos Efraín Pérez Rojas.” Native Networks/Redes Indígenas. Smithsonian National Museum of the American Indian. Obtenido el 12 de diciembre de 2011 en http://www.nativenetworks.si.edu/esp/rose/efrain_c_interview.htm\# open.

(2002). Re-imagining Politics: Video and Indigenous Struggles in Contemporary Bolivia. Tesis doctoral en antropología cultural. Dir.: M. Edelman. City University of New York. 